\title{
Du harcèlement sexuel sans conséquence pour la victime? Analyse discursive de la jurisprudence du Tribunal administratif du travail du Québec
}

Rachel Cox ${ }^{*}$ (i)

\begin{abstract}
Résumé
Cette étude s'intéresse au discours des juges du Tribunal administratif du travail du Québec en matière de harcèlement sexuel à partir d’un échantillon de 12 décisions rendues entre 2014 et 2017. Dans la moitié des décisions analysées, les motifs du juge reposent sur une logique binaire. Des binômes (femmes/hommes; subjectivité/ objectivité; émotionnalité/rationalité, etc.) se glissent dans l'appréciation de la preuve, perpétuant des mythes qui banalisent le harcèlement sexuel. Une logique binaire est généralement associée au rejet de la réclamation. En revanche, dans l'autre moitié des décisions, les motifs du juge prennent en compte le contexte ainsi que la complexité du harcèlement sexuel. Nos résultats illustrent comment le discours des juges sert soit à perpétuer, soit à remettre en question les stéréotypes négatifs relatifs au harcèlement sexuel des femmes au travail, et ce, dans un cadre juridique identique.
\end{abstract}

\begin{abstract}
This study looks at the discourse of Québec Labour Administrative Tribunal judges with respect to sexual harassment in a sample of 12 decisions rendered between 2014 and 2017. In half of the decisions, the reasons given by the judge for his or her decision are founded on binary logic. Assessment of the evidence is rife with binary thinking (women/men; subjectivity/objectivity; emotionality/ rationality, etc.) that perpetuates myths trivialising sexual harassment. Binary logic is usually associated with rejection of the claim. In contrast, in the other half of the decisions, the reasons given by the judge for his or her decision take into account context as well as the complexity of sexual harassment. Our results illustrate how, regardless of the fact that they are all applying the same law, judges' discourse can either perpetuate or challenge negative stereotypes about women who are victims of sexual harassment at work.
\end{abstract}

\footnotetext{
* L'auteure remercie Me Caroline Brodeur, LLM, pour sa relecture attentionnée de ce texte. L'auteure remercie aussi les évaluatrices, évaluateurs anonymes de cet article pour leurs suggestions judicieuses.
}

Canadian Journal of Law and Society / Revue Canadienne Droit et Société, 2019. This is an Open Access article, distributed under the terms of the Creative Commons AttributionNonCommercial-ShareAlike licence (http://creativecommons.org/licenses/by-nc-sa/4.0/), which permits non-commercial re-use, distribution, and reproduction in any medium, provided the same Creative Commons licence is included and the original work is properly cited. The written permission of Cambridge University Press must be obtained for commercial re-use.

Volume 34, no. 3, pp. 497-519. doi:10.1017/cls.2019.23 
Keywords: Sexual harassment, discursive analysis, legal remedy, law, language, feminism

\begin{abstract}
Discourse at its various levels is not mere talk, however; it is intimately connected to both thought and action. A way of talking about something is also a way of thinking about it, since what we say both reflects what we think and helps to shape what we and others will think in the future. And by structuring the way that actions get talked and thought about, discourse ultimately suggests and limits the possibilities for future actions. (Conley et O'Barr 1998, 18)
\end{abstract}

La légitimité du droit, et plus particulièrement celle des tribunaux, repose sur des valeurs d'impartialité et de neutralité ainsi que sur la règle de droit (Hunter 2015a). Dans cette conception traditionnelle du système juridique, le décideur doit faire preuve de détachement et d'impartialité : "This conventional judge is disembodied, objective, unemotional and impersonal. Law, fact and reason are the legitimate sources for judicial decisions... When applying law, personal views, biases, values and emotions should be set aside... " (Hunter, Roach Anleu et Mack 2016, 340).

La valeur de neutralité attribuée au droit porte à croire que celui-ci se situe en dehors de l'espace discursif et interprétatif. En réalité, toutefois, le droit est toujours sujet à interprétation (Bernheim 2013). La fonction juridictionnelle remplie par les tribunaux consiste à « dire » le droit pour disposer du litige. Chaque décision fournit un exemple de langage discursif dont l'analyse est susceptible de révéler les attitudes, les croyances, les valeurs, voire même parfois les émotions du décideur (Conley et O’Barr 1998).

Plus particulièrement, cet article s'intéresse aux décisions de la Division de la santé et de la sécurité du travail du Tribunal administratif du travail (TAT-DSST) qui portent sur l'admissibilité d'une réclamation pour une lésion professionnelle en lien avec le harcèlement sexuel. Nous commencerons par mettre en contexte le cadre juridique québécois du harcèlement sexuel en milieu de travail (1). Par la suite, nous exposerons la méthodologie de recherche adoptée (2) pour en venir à une présentation des résultats de notre analyse (3).

\title{
1. La Loi sur les accidents du travail et des maladies professionnelles, un recours qui élude les questions de genre
}

Sous le régime de la Charte des droits et libertés de la personne du Québec, le harcèlement sexuel au travail est considéré comme un phénomène de discrimination basé sur le sexe. En ce sens, une plainte pour harcèlement sexuel déposée en vertu de la Charte sera traitée dans un cadre juridique sensible au genre. Toutefois, depuis une quinzaine d’années, le Tribunal des droits de la personne du Québec rend de moins en moins de décisions sur le harcèlement sexuel au travail (Cox 2015). Ceci s'explique du fait que, d'une part, en cas d'atteinte à la santé, le recours exclusif visant à obtenir un dédommagement est celui de la Loi sur les accidents du travail et des maladies professionnelles (LATMP). D’autre part, il appert que la Commission des droits de la personne et des droits de la jeunesse dirige les victimes de harcèlement sexuel au travail vers le recours, en cas de harcèlement psychologique, prévu par la Loi sur les normes du travail (LNT) (Lippel, Cox et Aubé 2009, para 327). 
Le traitement du harcèlement sexuel sous l'angle du harcèlement psychologique a reçu une attention considérable (Cox 2014; CDPDJQ 2015), culminant dans l'adoption en juin 2018 de modifications législatives visant à faciliter l'accès au recours de la LNT pour les victimes de harcèlement sexuel en milieu de travail (Projet de loi 176, 2018). En comparaison, dans la dernière décennie, le processus d'indemnisation traitant le harcèlement sexuel comme un accident du travail en vertu de la LATMP a fait lobjet de moins détudes (voir toutefois Lippel et Demers 2000; Makela 2006).

Pourtant, en droit québécois, aux termes de la LATMP, la travailleuse dont la santé est atteinte par le harcèlement sexuel au travail est considérée avoir subi un " accident du travail ", soit « un événement imprévu et soudain, attribuable à toute cause, survenant à une personne par le fait ou à l'occasion de son travail et qui entraîne pour elle une lésion professionnelle » (Lippel et Cox 2010). Pour la période au cours de laquelle elle est atteinte de cette lésion professionnelle, la travailleuse ne peut poursuivre ni son employeur, ni son harceleur pour obtenir un dédommagement, car son recours exclusif pour des dommages matériaux, moraux et punitifs (Paquet 1997) ainsi que pour les soins psychologiques requis par son état, est une réclamation en vertu de la LATMP (Lippel, Cox et Aubé 2009). Il s'agit d'un régime sans égard à la faute qui offre une immunité civile à l'employeur (LATMP, art 438 et ss) ainsi qu'aux collègues de travail (LATMP, art 442). Aujourd'hui, le droit d'une travailleuse à un milieu de travail exempt de harcèlement sexuel se traduit plutôt par le droit à une indemnisation en vertu de la LATMP en cas de harcèlement sexuel, soit un cadre juridique qui élude complètement la question du genre ainsi que, plus largement, celle du droit à l'égalité des femmes (Langevin 2005).

Plusieurs auteures et auteurs ont émis des mises en garde contre les effets potentiellement néfastes de traiter le harcèlement sexuel de la même façon qu'un accident du travail. Entre autres, la réclamation pour un accident du travail implique un processus médico-légal (Lippel et Demers 2000) et une pathologisation des femmes qui en sont victimes, et ce, tant au plan matériel quau plan symbolique : "... not only do the victims of sexual harassment find themselves with an attenuated recourse, but all women are harmed by the law effectively saying: "If you have been harassed you must be sick" " (Makela 2006, 45).

Dans ce contexte juridique particulier, nous nous sommes intéressées au discours des juges du TAT-DSST lorsque ceux-ci interprètent des faits constituant du harcèlement sexuel.

\section{Considérations méthodologiques et pertinence de l'étude}

Le langage utilisé dans une décision juridique "peut simultanément refléter et renforcer les rapports de pouvoir » (Conley et O'Barr 1998, 14, traduction de l'auteure). Une analyse discursive est donc utile pour saisir comment des pratiques discursives contenues dans les décisions " reflètent, valident, et parfois remettent en question les hiérarchies sociales » (Conley et O’Barr 1998, 15, traduction de l'auteure) et, en particulier, la subordination des femmes aux hommes dans les milieux de travail. Pour exposer comment les rapports sociaux de sexe sont produits, entretenus ou contestés dans les décisions, nous avons procédé à une analyse discursive critique et féministe (Fairclough 2010; Livholts 2015; Lazar 2007). Une telle analyse vise à démasquer les «complex, subtle and sometimes not so subtle, ways in 
which frequently taken-for-granted gendered assumptions and hegemonic power relations are discursively produced, sustained, negotiated, and challenged in different contexts and communities" (Lazar 2007, 142).

D’une part, notre étude s'intéresse au pouvoir discursif du droit (Fudge 2013) et offre un cadre pour l'amélioration des pratiques argumentatives des juges de la TAT-DSST en matière de harcèlement sexuel, considérant ces pratiques à la fois comme « des phénomènes à être élucidées et des opportunités d'intervention - c'est-àdire des occasions de tenter de provoquer un changement social » (van Eemeren et al 1997, 16, traduction de l'auteure). D’autre part, alors que les analyses discursives s'intéressent souvent au micro-discours de différents individus ainsi quaux liens entre celui-ci et le macro-discours de la société, cette étude propose plutôt l'analyse d'un mezzo-discours, soit celui des décideurs d'un tribunal administratif. En élucidant comment ce mezzo-discours juridique reçoit, valide ou rejette le micro-discours des femmes harcelées, des harceleurs et des employeurs, létude illustre la relation dynamique entre différents niveaux de discours (Dougherty et Hode 2016).

Pour constituer notre échantillon, nous avons interrogé la base de données de SOQUIJ pour identifier les décisions rendues entre janvier 2014 et juin 2017 par le TAT-DSST (ou par son prédécesseur, la Commission des lésions professionnelles) qui portent sur des faits constituant du harcèlement sexuel. ${ }^{1}$ Ce dernier terme est entendu comme étant le harcèlement sexuel " donnant-donnant », soit, «[...] une conduite de nature sexuelle non sollicitée qui a un effet défavorable sur le milieu de travail ou qui a des conséquences préjudiciables en matière d'emploi pour les victimes du harcèlement » (Janzen c. Platy 1989, 1284).

Le terme inclut aussi le harcèlement sexuel résultant d'un " milieu de travail hostile », parfois appelé « harcèlement sexiste », ou harcèlement de genre, soit :

... une manifestation plus subtile, plus insidieuse que le harcèlement à connotations sexuelles. Ce type de situation ne comporte habituellement pas déléments qui pourraient laisser croire à une démarche de séduction. Au contraire, les paroles, les gestes ou les actes convergent vers la démonstration de l'inadéquation de la personne face à son milieu de travail, généralement à prédominance masculine, mais pas exclusivement non plus, et ce, simplement parce quelle est caractérisée par l'un des motifs énumérés à l'article 10 de la Charte; en l'occurrence, parce quelle est une femme... (Québec (CDPDJQ) 1998, para. 167-168)

Nous avons répertorié douze décisions portant sur des faits constituant du harcèlement sexuel ainsi défini, pour un total de 198 pages. Toutes les décisions sont

1 Cette recherche découle d'un plus grand projet de recherche portant sur le traitement du harcèlement sexuel alors que celui-ci est considéré, au plan juridique, soit comme du harcèlement psychologique, soit comme une lésion professionnelle, soit comme une violation de la Charte des droits et libertés de la personne (R. Cox, Conseil des recherches en sciences humaines, 20162018). Dans le cadre de celui-ci, l'auteure a repéré et, à l'aide du logiciel NVivo, codé les décisions pertinentes de lensemble des tribunaux concernés pour la période entre janvier 2014 et juin 2017. Déjà, après ce premier codage, l'auteure a remarqué qu'un langage binaire utilisé dans plusieurs décisions du TAT-DSST (et de son prédécesseur, la Commission des lésions professionnelles) distinguait le discours portant sur le harcèlement sexuel comme une " lésion professionnelle » du discours portant sur le harcèlement sexuel comme du " harcèlement psychologique " ou encore comme une violation de la Charte. Lauteure a donc entrepris une analyse discursive plus approfondie du discours des décideurs du TAT-DSST, doù le présent article. 
publiques et disponibles sur l'Internet. Sept décisions de notre échantillon ont été caviardées par le tribunal. Six réclamations ont été acceptées alors que six autres ont été rejetées. ${ }^{2}$ Ce sont des femmes qui ont déposé la réclamation dans onze décisions sur douze; il ne s'agit d'un homme que dans une décision. Tous les harceleurs étaient des hommes.

Nous avons analysé les décisions à l'aide du logiciel de recherche qualitative NVivo 11. Adoptant une démarche inspirée de la théorisation ancrée (Corbin et Strauss 2008), lors d'un codage de premier niveau (open coding), nous avons d'abord identifié des catégories descriptives telles que « description de la personne plaignante ", " conséquences du harcèlement ", " crédibilité », « dénonciation ", " sentiments », " perceptions », et ainsi de suite. Ensuite, à partir de ces catégories descriptives, lors d'un codage de deuxième niveau (axial coding), des catégories analytiques ont « émergé » (Corbin et Strauss 2008, 6), dont une série ayant comme fil conducteur un langage imbibé de logiques binaires : victime/agresseur, objectivité/ subjectivité, crédible/non crédible, perception/comportement, sentiments/réalité et ainsi de suite. Ensuite, pour replacer dans leur contexte les extraits analysés, toutes les décisions ont été relues intégralement. Cette relecture a permis de scruter de nouveau les décisions, à la fois pour déceler l'ensemble des logiques binaires présentes (selective coding) et pour identifier des contre-exemples de décisions exemptes de logique binaire, soit une stratégie de validation impliquant la recherche active de cas négatifs (disconfirmation) (Corbin et Strauss 2008, 84).

\section{Résultats : la logique binaire}

Lors de l'analyse des décisions, les catégories analytiques retenues avaient pour fil conducteur un langage binaire ou bien, en revanche, une prise en compte du contexte et de la complexité du harcèlement sexuel. Or, une critique féministe du droit de longue date souligne que la pensée occidentale est structurée autour d'une série complexe de binômes dont un pôle, considéré supérieur, est associé aux hommes (rationnel, actif, objectif, abstrait, logique, culture...) alors que l'autre, associé aux femmes (irrationnel, passif, contextualisé, émotif, nature...) est considéré inférieur (Olsen 1998, 691).

En effet, une logique binaire positionne deux concepts comme étant opposés alors que, dans les faits, ils ne le sont pas. L’un des pôles du binôme est plus valorisé que l'autre : "Binary pairs such as male/female, gay/straight, work/life and whitel black serve to reify power by demarcating particular boundaries that are almost always hierarchically structured. As a naturalized system of thought, binary logic perpetuates power differentials and the systemic subjugation of those constituted as "other" ". [Références omises.] (Dougherty et Hode 2016, 1733).

Le binôme émotionnalité/rationalité illustre comment, dans les pratiques organisationnelles, différents binômes s'allient avec le binôme du genre pour placer les hommes dans une position structurelle dominante :

2 Dans cinq des six réclamations acceptées, le décideur était une femme. Dans trois des six réclamations rejetées, le décideur était une femme. Toutefois, compte tenu de la petitesse de notre échantillon, toute conclusion concernant une association entre le genre du décideur et les chances de succès d’une réclamation en lien avec le harcèlement sexuel serait prématurée. 
...emotionality in organisations is tainted and stigmatized. Yet, rationality/ emotionality represents a false binary in that rationality is not the opposite of emotionality. In fact, rational thought requires emotional engagement. As such, emotionality and rationality are inextricably intertwined in organizations. Yet, these terms have been framed in organizational practice as an oppositional binary pairing with rationality associated with masculinity and emotionality associated with femininity. [Références omises.] (Dougherty et Hode 2016, 1734)

La logique binaire joue un rôle important dans l'argumentation juridique. Les exemples de binômes, ou de dichotomies, sont nombreux dans le domaine juridique : légal/illégal, droit/obligation, apte/inapte, etc. (Reiner 2016). Par ailleurs, les dichotomies ne sont pas pour autant " un impératif de la nature du droit » (Reiner 2016, 427). Comme le binôme émotionnalité/rationalité, certaines dichotomies du droit sont fausses : «L'inconvénient des classifications binaires, c'est qu'elles induisent à des simplifications, là où le critère de distinction n'est pas de nature à garantir l'exclusion mutuelle des catégories ou lorsqu'il existe encore d'autres catégories appartenant à l'extension du terme générique ou lorsqu'il existe même un continuum entre les deux pôles (fausses dichotomies) » (Reiner 2016, 457).

De plus, les dichotomies du type " passe-partout » (objectif/subjectif; cause/effet; actif/passif, et ainsi de suite) remplissent une fonction rhétorique et tendent à transporter des stéréotypes : "Par leur notoriété et leur familiarité, ces dichotomies profitent d'une plausibilité inhérente, renouant avec des intuitions de la pensée en langage normal, et se passent de justification... Comme toutes les dichotomies, les dichotomies passe-partout laissent croire qu'elles sont complètes et qu'elles couvrent tout le champ des possibles » [Références omises] (Reiner 2016, 457).

Au-delà de l'acte de classification inhérent à l'application du droit, nous avons été à même de constater que les décisions du TAT-DSST sont susceptibles de faire appel à des dichotomies suspectes, ou en d'autres termes, à un langage binaire, soit une forme de classification - et une façon de penser - dans laquelle les femmes et les caractéristiques associées aux femmes sont infériorisées (Olsen 1998; Dougherty et Hode 2016).

Plus spécifiquement, dans la moitié des décisions analysées, nous avons observé le déploiement d'une succession de binômes : comportement/perception; objectivité/subjectivité; faits/fabulations; crédibilité/absence de crédibilité; réalité/ sentimentalité; rationalité/émotivité; sphère publique/sphère privée; normalité/ déviance, pour ne nommer que ceux-là. Voir le Tableau I.

Le binôme du genre (femmes/hommes) constitue la charpente sur laquelle tous les autres binômes se greffent. Même s'il est associé à quatre des six réclamations qui sont rejetées, ce langage binaire peut être présent même quand la réclamation est acceptée (deux réclamations sur six). Et, même si un langage non binaire est associé à l'acceptation de la réclamation dans quatre décisions sur six, l'absence de langage binaire ne signifie pas nécessairement que la réclamation sera acceptée. Voir Figure 1.

À titre d'illustration, la décision M.N. et Compagnie A (2016 QCTAT 5227) traite de la réclamation d'une travailleuse de la construction harcelée sexuellement 
Tableau I:

Binômes présents dans les décisions analysées

\begin{tabular}{ll}
\hline Masculinité & Féminité \\
\hline Comportement & Perceptions \\
Objectifs & Subjectives \\
Faits & Fabulations \\
Crédibles & Exagèrent/Non crédibles \\
Réalité & Sentimentalité \\
Rationalité & Émotionnalité \\
Sphère publique & Sphère privée \\
Normalité & Déviance \\
Victimes des plaintes pour harcèlement sexuel & Agresseurs - déposent des plaintes par vengeance, \\
& jouent à la victime \\
Comportement contraignant & Comportement contrariant \\
Harcèlement sexuel avec conséquence & Harcèlement sexuel sans conséquence \\
\hline
\end{tabular}

et agressée au travail à plusieurs reprises, au point où, souffrant d'un trouble d'adaptation, elle constate qu'elle n'est plus capable d'exercer son métier dans le domaine de la construction. La juge emploie un langage binaire pour décrire son témoignage : "À l'audience, la travailleuse témoigne de façon assurée et crédible, sans exagération, malgré quelques épisodes émotifs rapidement maîtrisés " (nos italiques).

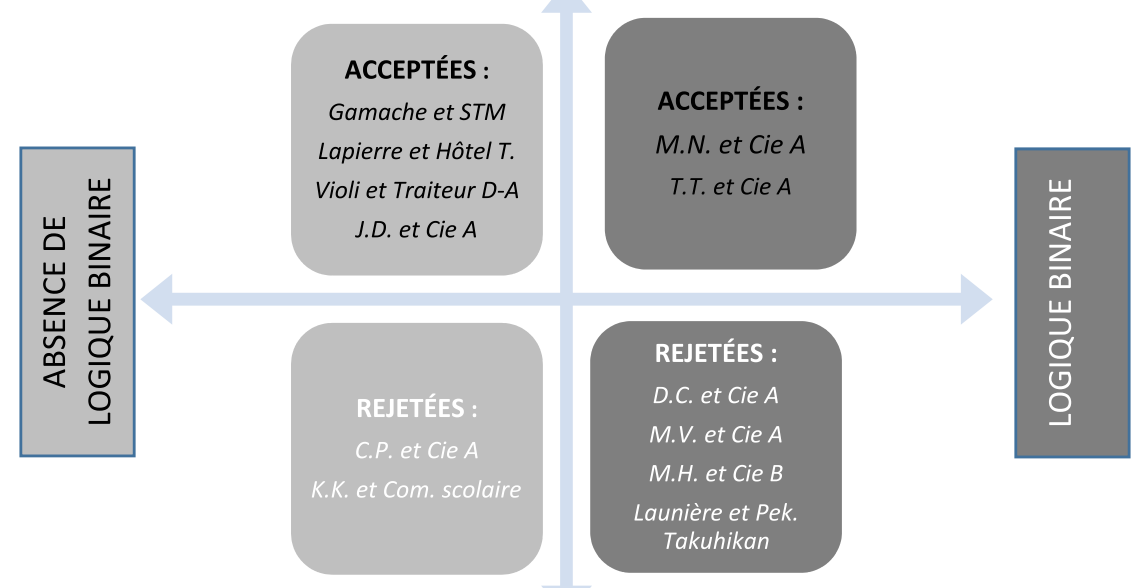

\section{Langage binaire}

Figure 1 Répartition des décisions selon le sort de la réclamation et la présence de logique binaire 
La juge conclut que la travailleuse est crédible, ou rationnelle, mais... en dépit de ses émotions : " malgré quelques épisodes émotifs ». Ce langage rappelle le binôme émotionnalité/rationalité, indiquant que la travailleuse peut être crue, mais uniquement parce qu'elle est parvenue à maîtriser les émotions qui autrement auraient pu l'amener à exagérer. Au faux binôme émotionnalité/rationalité se greffe une variante : le binôme crédibilité (émotions maîtrisées)/absence de crédibilité (émotivité).

Dans cette même cause, l'employeur ne conteste pas les événements rapportés. De plus, le témoignage d'un collègue de travail corrobore l'agression et le harcèlement vécus par la travailleuse sur le chantier. La juge conclut que : "Ces deux témoignages [ceux de la travailleuse et de son collègue] sont suffisamment probants pour établir qu'il s'agit bien de faits, et non de perceptions ou de fabulations, et ces faits nont pas été contredits. Le Tribunal considère cette preuve prépondérante ».

Ici encore, un langage binaire crée une fausse opposition entre les perceptions (subjectives) de la travailleuse et les faits (objectifs) entourant le comportement du harceleur, alors qu'il est facile de concevoir un rapport étroit entre ceux-ci : « il s'agit bien de faits, et non de perceptions ». Les deux témoignages sont considérés " suffisamment probants ", nous avise la juge, rappelant l'enjeu de la crédibilité. De surcroît, le même passage de la décision associe des "perceptions " à des « fabulations ", renforçant encore plus l'absence de légitimité de la perception en matière de harcèlement sexuel. La perception qu'a la femme harcelée de la réalité qu'elle vit est dépeinte comme suspecte, problématique, située à l'opposé des faits, c'est-à-dire au comportement - factuel, objectif - de l'homme, supposé harceleur.

Dans cet exemple, le critère juridique d'une preuve qui va « au-delà des perceptions » de la travailleuse constitue le point de chute pour ce langage binaire. Le harcèlement sexuel est tellement évident que, comme l'exception qui prouve la règle, la réclamation est acceptée, et ce, en dépit des motifs du juge qui véhicule des valeurs sexistes et des préjugés concernant les femmes qui rapportent un harcèlement sexuel. Toutefois, signalons qu'il est possible pour un décideur de se référer à une preuve qui va " au-delà des perceptions » sans que celui-ci ne dérape vers un rejet de la subjectivité du harcèlement, devenant ainsi un « lieu d'argumentation » (Reiner 2016, 453) plutôt qu'un critère juridique. Pour illustrer, dans l'affaire Violi et Traiteur Dine-Aire (2015 QCCLP 6867), la juge rapporte la preuve sur huit pages et ensuite, annonce succinctement:

Le tribunal est d'avis que la preuve prépondérante démontre des propos inappropriés, injurieux, abusifs et à caractère sexuel à l'endroit de la travailleuse. Lattitude, les propos et comportements de monsieur Katz [le harceleur] constituent une série d'événements qui peuvent être qualifiés d'imprévus et soudains, survenus par le fait du travail. La preuve démontre des éléments objectifs, corroborés, qui vont au-delà de la perception des événements qu'a pu avoir la travailleuse et qui débordent largement le cadre normal dans un milieu de travail.

\section{Inversement des rôles : la victime devient celle qui harcèle}

Dans deux décisions où la réclamation est acceptée, le discours des juges est empreint d'un « paternalisme ambivalent » (Nightingale, Quayle et Muldoon. 2017, 137). 
Par exemple, la décision T.T. et Compagnie A (2014 QCCLP) traite de la réclamation pour un trouble d'adaptation diagnostiqué chez une travailleuse dans le domaine de la vente ayant subi du harcèlement psychologique et du harcèlement sexiste de la part d'un compétiteur. Dans la présentation des motifs de la décision, et en l'absence de preuve contradictoire à ce sujet, le juge affirme d'abord que : «...la preuve ne démontre aucunement que la travailleuse ait eu un comportement ou une attitude inappropriée à l'endroit de monsieur M. [le harceleur] ».

Ici, le juge explore l'inversion des rôles : le harceleur ne serait-il pas la victime, et la travailleuse, l'assaillante? Le binôme victime/harceleur est inversé. Ensuite, le juge note que la supérieure immédiate de la travailleuse n'a « formulé aucun reproche à l'endroit du travail effectué par la travailleuse, qualifiant celle-ci de très bonne vendeuse ». Or, le rendement de la travailleuse n'est aucunement en litige. Mais en faisant cette remarque, le juge évoque un autre binôme, soit celui de la déviance/ normalité, en l'espèce la conformité de la travailleuse aux attentes de son patron.

Dans le même ordre d'idées, le juge se réfère à deux reprises à l'expertise psychiatrique produite par la travailleuse pour conclure que le témoignage de celle-ci est crédible, puisque la psychiatre décrit la travailleuse comme « nétant pas agressive, théâtrale, revendicatrice, méfiante ou interprétative. " En insinuant que la travailleuse (crédible) doit être distinguée des femmes (non crédibles) qui sont méchantes ( " agressive » ou « revendicatrice »), irrationnelles ( "théâtrale » ou " interprétative ») et émotivement instables ( "méfiante»), le juge fait appel de nouveau au binôme émotionnalité/rationalité pour discréditer les femmes, tout en justifiant sa décision d'accepter la réclamation de la travailleuse devant lui. Ce faisant, il perpétue le mythe - ou en d'autres termes, le macro-discours - voulant que des femmes émotives et irrationnelles se plaignent de harcèlement sexuel sans justification (Lonsway, Cortina et Magley 2008), ce qui justifierait un scepticisme à l'égard de leur témoignage comme seul moyen de preuve. La logique discursive employée par le juge se réfère de nouveau au binôme de la déviance/normalité, car, par une logique contraire, en l'espèce, le juge associe la crédibilité de la travailleuse au caractère conciliant, modeste, passif, crédule et naturel de celle-ci, bref, à sa conformité à des traits associés aux stéréotypes de la féminité.

\section{Perception (émotion) v. Comportement (rationalité)}

Par ailleurs, dans quatre décisions où la réclamation est rejetée, le langage binaire employé est sans équivoque. À titre d'illustration, la décision Launière et Pekuakamiulnuatsh Takuhikan (2016 QCTAT 4968) met en cause une travailleuse occupant un emploi de policière pour une communauté autochtone. Le juge oppose directement la perception émotive de la travailleuse qu'elle subit $\mathrm{du}$ harcèlement sexiste à titre de femme policière au comportement rationnel de ses collègues masculins.

De retour au travail après plusieurs années de congé de maladie et de maternité, la policière intègre un milieu de travail où les rapports sont tendus notamment en vue de la négociation du renouvellement de la convention collective. Un conflit syndical oppose les policiers détenant un poste permanent, dont la travailleuse, qui est la seule femme dans ce groupe, à ceux détenant un poste temporaire. Le retour 
au travail de la travailleuse entraîne une modification des équipes de patrouille déjà formées. Dès son arrivée, le superviseur de la travailleuse l’informe que certains de ses collègues sont d'avis qu'elle n'est " pas prête " pour un retour au travail comme patrouilleur et qu'il sera difficile de trouver quelqu'un qui acceptera de travailler avec elle. Le juge reconnait les « difficultés qu'elle [la travailleuse] rencontrait constamment avec certains collègues " mais conclut néanmoins que «... La preuve ne révèle aucunement la présence d'un geste direct posé contre la travailleuse, si ce n'est qu'un climat d'insécurité prévaut, lui-même à l'intérieur d'un climat syndical malsain en 2014, de sorte que la perception de la travailleuse peut être grandement amplifiée ».

La prétention de la travailleuse que son milieu de travail lui était hostile est écartée à titre de " perception » qui a pu « être grandement amplifiée » par le « climat d'insécurité " général et le « climat syndical malsain ». Si des perceptions subjectives ou des émotions sont opposées à la rationalité objective et crédible, des perceptions amplifiées par des sentiments d'insécurité le sont encore plus. Plusieurs binômes - subjectivitélémotionnalité/absence de crédibilité versus objectivité/rationalité/crédibilité s’amassent.

Souffrant d'un trouble d'adaptation, la travailleuse allègue divers incidents pour démontrer à quel point son milieu de travail était hostile. Lors de l'un de ces incidents, un collègue lui saute dessus sans préavis pour lui faire une clé de bras, soit une prise au haut du corps. Le juge déclare que : "Dans le contexte de son retour au travail et des difficultés quelle rencontrait constamment avec certains collègues, le Tribunal est convaincu que la travailleuse n’a pas trouvé l'événement drôle et n'a pas jugé qu'il s'agissait d'un jeu. Elle a réellement senti une agression physique en raison des agissements inappropriés de son collègue » [nos italiques].

Le juge note que la travailleuse ne s’attendait pas à l'agression, ne la désirait pas, a demandé à deux reprises à son collègue de la lâcher et, face à son refus, a dû forcer pour se dégager de la prise. Rappelons que le tout se situe dans un contexte où ses collègues masculins remettent ouvertement en question la capacité de la travailleuse à titre de policière. Toutefois, le juge écarte cet incident à titre dévénement dont le cumul avec d’autres événements pourraient constituer un événement imprévu et soudain au sens de la Loi :

Cela étant précisé, le Tribunal est d'avis que la preuve démontre cependant qu'il s'agit d'un événement isolé dans un contexte, certes conflictuel, mais qui n'impliquait pas une agression physique réelle contre la travailleuse, car d’autres policiers ont reçu ou pratiqué cette prise entre eux.

Alors, même si la travailleuse «a réellement senti une agression physique», pour le juge, lévénement «n'impliquait pas une agression physique réelle ». La travailleuse est un témoin non crédible de sa propre expérience car, de bonne foi, elle ressent des choses qui en réalité n'existent pas. Pour reprendre Catharine MacKinnon, la conclusion du juge est : "Not that the acts did not occur, but rather that it was unreasonable to experience them as harmful» $(1979,108)$.

L'exercice du pouvoir d'interprétation de la preuve par le juge produit littéralement la réalité (Conley et O’Barr 1998, 9) dans un cadre d’analyse juridique 
masculin et hégémonique, et ce, à l'aide d'un langage binaire multidimensionnel : émotionnalité/rationalité; perception/comportement; crédibilité/absence de crédibilité, faiblesse/force, incapacité/capacité, fermme/homme, et finalement... sentiments/réalité.

Cette décision illustre à la fois le caractère réducteur du langage binaire (Reiner $2016,451)$ et comment celui-ci agit au détriment d'une approche contextuelle dans laquelle on tient compte des rapports sociaux entre les personnes pour déceler le harcèlement sexiste.

\section{Colère, donc vengeance?}

Même si le binôme femmes/hommes informe, traverse et transcende les autres binômes, il se décompose également. Par exemple, parmi les émotions, la colère est perçue comme étant une émotion masculine de force et d'autonomie, alors que, face au harcèlement sexuel, on attend des femmes qu'elles se sentent désemparées, désespérées, bouleversées, c'est-à-dire qu’elles éprouvent des émotions féminines de dépendance (Conley et O’Barr 1998, 121-122). Dans la décision M. V. et Compagnie A (2014 QCCLP 1981), la travailleuse est commis-caissière dans un dépanneur de quartier fréquenté par les parents et amis de tous les protagonistes. La travailleuse allègue des commentaires, gestes et attouchements à caractère sexuel de la part du gérant :

Par la suite souvent il venait vers moi en ce touchant partout et se sortait la langue comme un dansseur nue. Une fois même devant un livreur de biere alors qu'il était a côté de moi derriere le comptoir caisse il s'est leve dans les airs et a ouvert ses jambes, une jambe sur chaque comptoir comme si il voulais que je lui fasse une fellation. Encore la je passe pour quoi devant les gens. Je ne me rappelle pas ce que je lui ai dit mais le livreur ma regarder et m'a dit «Elle est pas contente la madame » c'est sur voyon donc souvent il venais se coller de côter et passait sa main dans mon dos jusquà la hanche, parfois même devant les clients, assez que y'en avais quelque uns qui croyais que cétais mon chum. Voyons donc, même une autre fois alors que mon conjoint était la en train de se faire un caffé, il est passé derriere moi et ma carresser les cheveux, il ne devais surement pas avoir vu mon conjoint...

Un jour, le gérant prend la travailleuse de force par derrière, en gémissant sur un ton obscène à son oreille; la travailleuse dénonce ce comportement à son employeur. Sauf qu'en retournant au travail le lundi suivant, la travailleuse constate quelle est ostracisée par la parenté du gérant qui fréquente le dépanneur. Désemparée, elle quitte son emploi. Un médecin diagnostique la travailleuse comme souffrant d'un « stress post-traumatique post-agression » et la met en arrêt de travail. Elle ne retournera plus à son emploi.

Or, avant même d'entamer l'analyse du cadre juridique de la réclamation, la juge rompt avec la séquence classique utilisée dans les décisions du tribunal (les règles de droit, les faits mis en preuve, l'application des règles de droit aux faits) pour souligner la colère, voire la haine de la travailleuse :

À l'audience, le tribunal comprend cependant un élément important. La travailleuse est en colère parce que monsieur C... a visiblement raconté les faits différemment à son frère et à sa nièce puisque ces derniers ne saluent pas la travailleuse lors de leur visite du 22 avril 2013. C’est à 
compter de ce jour et de cette réaction que la travailleuse décide de déposer une plainte à la police.

Avant cette date, la travailleuse avait manifesté son mécontentement à son employeur, probablement à juste titre, contre les gestes de monsieur C....

À l’audience, la travailleuse exprime sa haine envers monsieur C... en raison duquel elle allègue avoir perdu son emploi.

D’une part, le langage dépeint la travailleuse comme étant irrationnelle et émotivement instable, voire méchante, car poussée par sa haine envers le harceleur, elle dépose une réclamation à la CSST et porte plainte à la police. Passant par le binôme émotionnalité/rationalité, le juge finit par inverser le binôme victime/harceleur, suggérant que c'est la travailleuse qui est en train d'attaquer le harceleur.

Effectivement, la juge poursuit son analyse et, au soutien de sa conclusion que la travailleuse a déposé une réclamation dans un esprit de vengeance, invoque le fait que la travailleuse a longtemps toléré le harcèlement sexuel du gérant : "Le tribunal estime que c'est néanmoins la réaction du frère et de la nièce de monsieur C... qui est à l'origine de la réclamation de la travailleuse. Cette dernière semblait avoir trouvé le moyen de gérer le comportement de monsieur C... en le dénonçant à sa face même et en dénonçant son comportement à son employeur ».

Selon la juge, en réalité, la travailleuse est simplement en colère envers le harceleur pour avoir monté des membres de sa parenté contre elle. Elle ne fait que « jouer à la victime ». En tant que femme colérique et amère, donc peu féminine (la décision reproduit même les injures prononcées envers le harceleur), sa probité est compromise. Et puisquelle a déjà enduré le harcèlement sexuel un certain temps sans faire de dénonciation formelle, selon la juge, celui-ci ne saurait être relié à l'atteinte à sa santé mentale, la preuve en est qu'elle semble « avoir trouvé le moyen de gérer le comportement de monsieur C...». Avec pareille représentation de la situation, la victime ne s'en sort pas. Aucun moment n'est le bon pour dénoncer le harcèlement sexuel! À la limite, ce raisonnement insinue que le simple fait de déposer une réclamation représente en soi un geste suspect. Le tout évoque les mythes voulant que les femmes qui tardent à déposer des plaintes pour harcèlement sexuel soient probablement en train d'inventer les faits dénoncés de toute pièce, ou encore que le dépôt tardif d'une plainte implique nécessairement des faits qui, au final, ne sont pas assez graves pour être considérés comme étant du harcèlement sexuel (Lonsway, Cortina et Magley 2008, 600).

Et puis, plus loin dans la décision, la juge affirme de nouveau que :

... le tribunal ne croit pas que la travailleuse a craint pour son intégrité physique par les gestes déplacés de monsieur $\mathrm{C}$... ou par la réaction de son frère et de sa nièce.

Les gestes décrits ne semblent pas avoir provoqué de peur chez la travailleuse, mais de la colère. (...)

Et en dépit du fait que le médecin ait mis la travailleuse en arrêt de travail en raison d'un stress post-traumatique, puisque celle-ci était retournée au travail le lundi suivant l'agression du vendredi précédent, la juge conclut qu'elle ne s'est pas vraiment sentie menacée : «D’ailleurs, la preuve révèle que la travailleuse est retournée 
au travail le 22 avril 2013. Elle ne se sentait donc pas menacée à la suite des gestes de monsieur C....»

Lors de l'audience, la travailleuse est interrogée concernant la prise des antidépresseurs qui lui ont été prescrits par le médecin ; elle dira « en avoir pris aléatoirement et les avoir cessés sans suivi médical ». La juge note également que la travailleuse n'a pas entrepris la psychothérapie pourtant recommandée de façon urgente par son médecin. Pour la juge, le tout accréditant la thèse selon laquelle les conséquences du harcèlement sexuel ont été minimes pour la travailleuse.

La juge conclut que « les gestes décrits quant aux agissements de monsieur C... le 19 avril 2013 et lors dévénements antérieurs ne revêtent pas le degré de gravité propre à engendrer un stress post-traumatique ». Constatant l'absence de relation entre le diagnostic et les événements allégués, la juge rejette la réclamation de la travailleuse, et ce, même si la juge affirme que les gestes du harceleur étaient « répréhensibles » et débordaient le " cadre normal de travail auquel la travailleuse serait en droit de s'attendre ». Pour reprendre MacKinnon de nouveau, en rejetant la réclamation, la juge conclut, "Not that the acts did not occur, but rather that it was unreasonable to experience them as harmful » $(1979,108)$. Le langage discursif de la juge mobilise le cadre juridique de la LATMP pour légitimer un point de vue voulant que le harcèlement sexuel fût, à toutes fins pratiques, sans conséquence pour la travailleuse.

Dans la décision M.H. et Compagnie B (2016 QCTAT 3846), la travailleuse présente une réclamation pour un trouble d'adaptation en lien avec du harcèlement psychologique et sexuel de la part d'un collègue. Peu de temps après un événement culminant lors duquel le collègue en question crie après la travailleuse et lance une tasse dans sa direction, elle consulte un médecin. Elle obtient sans délai un transfert dans un autre établissement de l'employeur sans être obligée d'arrêter de travailler. En fait, même si la travailleuse se dit traumatisée et médicamentée depuis cet événement, elle ne cessera de travailler que deux ans et demi plus tard. Or, habituellement, un trouble d'adaptation se dissipe lorsque le facteur de stress n’est plus présent, se résorbant généralement dans un délai de six mois (APA 2000). Sa réclamation est rejetée, puisque «le Tribunal ne peut conclure à une relation probante avec les diagnostics en cause ». Cela n'a peut-être rien de surprenant, compte tenu de la chronologie des événements rapportés.

Néanmoins, la juge mobilise un langage binaire pour justifier sa décision et notamment, son appréciation de la crédibilité de la travailleuse; la tendance à parler fort de la travailleuse et de son harceleur (allégué); et son attribution de la cause de l'incapacité de la travailleuse à un conflit interpersonnel « ayant mal évolué ».

\section{L’appréciation de la crédibilité de la travailleuse}

Dans M.H. et Compagnie B, à la demande de son médecin traitant, la travailleuse est évaluée par un psychiatre. Dans son rapport, le psychiatre mentionne que la travailleuse présente des « éléments de personnalité histrionique ». ${ }^{3}$ Faisant fi des

Pour une critique de la médicalisation des victimes de harcèlement sexuel, voir Finn Makela, " "Tell Me Where It Hurts": Workplace Sexual Harassment Compensation and The Regulation of Hysterical Victims », (2005-2006) 51 McGill Law Journal 27. Voir aussi Cox 2014. 
règles de preuve (et profitant peut-être du fait que le représentant de la travailleuse n'est pas un avocat), la procureure de l'employeur saisit cette ouverture pour administrer elle-même une preuve au sujet de la personnalité histrionique :

Pour compléter la preuve, la procureure de l'employeur dépose un extrait du site de la Fondation des maladies mentales (E-2) concernant particulièrement la personnalité histrionique, qui serait plus souvent diagnostiquée chez les femmes. On y décrit notamment le mode général de réponses émotionnelles excessives que ce genre de personnalité peut apporter et leur quête d'attention, pouvant commencer au début de lâge adulte et se présenter dans une série de contextes. On cite en exemple leur inconfort dans les situations où ces individus ne sont pas le centre de lattention, leur interaction avec les autres pouvant souvent être caractérisée par une séduction sexuelle inappropriée ou un comportement provocateur, leur expression émotionnelle superficielle et rapidement changeante, qu'ils peuvent utiliser régulièrement leur apparence physique pour attirer l'attention sur eux, leur manière de parler trop subjective mais pauvre en détails, qu'ils montrent de l'auto-dramatisation, de la théâtralité et une expression exagérée d'émotions, de leur suggestibilité et du fait qu'ils sont facilement influencés par autrui ou par les circonstances et qu'ils peuvent considérer les relations plus intimes quelles ne le sont en réalité. [Nos italiques]

Outre les traits de personnalité dite « histrionique » invoqués par le psychiatre de la travailleuse, l'extrait déposé en preuve par la procureure de l'employeur introduit sournoisement d'autres manifestations de la personnalité histrionique, soit:

- Inconfort dans les situations où ces individus ne sont pas le centre d'attention.

- L'interaction avec les autres est souvent caractérisée par une séduction sexuelle inappropriée ou un comportement provocateur.

- Utilisent régulièrement leur apparence physique pour attirer l'attention sur eux.

- Considèrent les relations plus intimes qu’elles ne le sont en réalité.

D’emblée, ces traits rappellent plusieurs préconçus négatifs, dont le fait que les femmes qui portent plainte pour harcèlement sexuel le font pour attirer l'attention, qu'elles exagèrent, et le fait qu'après avoir entretenu un fantasme de relation intime avec un homme au travail, il arrive que certaines femmes imaginent que celui-ci les a harcelées (Lonsway, Cortina et Magley 2008, 600). Dans cette décision, la juge va se référer à plusieurs reprises au rapport du psychiatre pour souligner le manque de crédibilité de la travailleuse, sa " propension à dramatiser les événements ", la disproportion de sa réaction au harcèlement pour finalement conclure que ses réactions sont non seulement disproportionnées, mais anormales : «[... Tout comme le [psychiatre], on remarque certaines réactions plutôt disproportionnées, voire anormales, chez la travailleuse ».

La juge souligne la suggestibilité de la travailleuse - l'un des traits de la personnalité histrionique - pour laisser entendre que celle-ci exagère :

Elle a été dirigée à l’organisme Viol Secours par son CLSC. Grâce au soutien de cet organisme, elle a pu réaliser qu'elle souffrait de tous les symptômes d'une victime d'agression. 
... la travailleuse décrit certains contacts physiques inappropriés avec monsieur G..., qu'elle vient à qualifier d'attouchements sexuels. Elle se tourne éventuellement vers Viol Secours pour obtenir de l'aide... elle va en venir à qualifier monsieur G... d'agresseur et [à] faire des rapprochements entre sa situation et certains dossiers fortement médiatisés.

Le langage de la juge dépeint la travailleuse comme étant irrationnelle et émotivement instable, ce qui donne à ses perceptions un caractère biaisé et erroné. Passant par le binôme émotionnalité/rationalité, la juge inverse de nouveau le binôme victime/harceleur, suggérant que c'est la travailleuse qui est en train d'attaquer le harceleur en qualifiant des contacts physiques inappropriés d'attouchements sexuels et en traitant celui-ci - «monsieur G... », pour la juge - « d'agresseur ». Encore une fois, pour la juge, la travailleuse « joue à la victime », se réclamant d'un mouvement de femmes victimisées alors que, toujours selon la juge, elle n’en est pas une.

Chaque fois que la travailleuse se réfère à la notion de harcèlement sexuel, la juge prend ses distances :

(...) la travailleuse dénonce principalement un comportement plutôt colérique de monsieur G... à son égard, dont un esclandre lui ayant valu une suspension d'une semaine, et des contacts physiques inappropriés, qu'elle qualifie d'attouchements sexuels.

(...) la travailleuse décrit certains contacts physiques inappropriés avec monsieur G..., qu'elle vient à qualifier d'attouchements sexuels.

De plus, la juge persiste à caractériser le harcèlement dont la travailleuse se plaint comme une tentative de séduction de la part de son collègue. Or, la travailleuse se plaint plutôt du caractère hostile du comportement du harceleur depuis quelle a repoussé des avances subtiles de sa part. Même, selon le harceleur, c'est plutôt la promotion qu'a obtenue la travailleuse qui déclenche une détérioration de leur relation : "Selon monsieur G..., leur relation se détériore à partir du moment où la travailleuse devient superviseure à l'établissement 5050. Selon lui, la travailleuse a tendance à parler très fort, ce qui l'irrite et le fait réagir émotivement ».

Néanmoins, la juge persiste à employer des termes de sentimentalité et de séduction pour interroger le comportement de celui-ci à l'égard de la travailleuse : "Questionné sur ses intentions sentimentales, voire affectives, envers la travailleuse, monsieur G... assure qu'il n’en a aucune. À lépoque, il venait de se séparer de sa conjointe. Il ne voulait aucunement fréquenter la travailleuse ni personne d'autre ».

Ce faisant, la juge adopte une approche qui normalise le harcèlement sexuel plutôt que de le concevoir comme une intrusion (Wood 2005). Pourtant, dans le domaine des droits de la personne, «À l'instar de la doctrine, la jurisprudence reconnaît que la véritable finalité des comportements prohibés n’est pas la séduction; les comportements visent essentiellement à garder les femmes, tant individuellement que collectivement, enfermées dans une position d'infériorité » (Québec (CDPDJQ) 1998, para. 148).

\section{Double standard}

Dans M.H. et Compagnie B, la supérieure de la travailleuse et de son harceleur les décrit chacun comme étant un employé apprécié. Toutefois, les deux ont un défaut : ils 
parlent fort. Ce défaut est présenté dans des termes plus cléments pour le travailleur que pour la travailleuse. Lui, "sa voix porte ", il a un " caractère », tandis qu'elle, « on » dit d’elle « qu'elle a parfois tendance à crier» :

Jusqu'en 2012, la dynamique entre les employés est bonne, selon madame M.... Selon ses observations, monsieur G... peut être parfois émotif. Il peut pleurer comme il peut se fâcher, selon les circonstances. Sa voix porte, surtout en cuisine.

Madame M... dit avoir une bonne confiance en la travailleuse. Elle a une bonne relation avec elle. La travailleuse a également une bonne relation avec les autres employés, quoique l’on dise quelle a parfois tendance à crier.

À trois reprises, la juge revient sur l'idée que la travailleuse " parle fort » ou " crie ", ce qui «agace » le harceleur : «... Monsieur G... est agacé par le fait que la travailleuse a tendance à parler fort, voire même crier, ce qui l'indispose. Monsieur G... peut donc démontrer de l'impatience et de l'irritation. C'est ce qui peut expliquer son comportement parfois colérique à légard de la travailleuse ».

La juge rapporte-t-elle simplement les témoignages? Ou relate-t-elle la preuve avec une intention annonciatrice de sa propre appréciation de celle-ci? Il semblerait que ce soit le dernier cas, car la juge introduit les motifs de sa décision par la déclaration suivante : « Monsieur G... travaille à l'établissement 5050 depuis 2003. Il y travaille encore. Et, à partir de la preuve soumise, le Tribunal comprend qu'il est apprécié des employés et des propriétaires, malgré son caractère ».

Or, le fait que le harceleur soit apprécié dans son milieu de travail ou ses états de service ne sont aucunement pertinents à la question, à savoir si sa conduite envers la plaignante était vexatoire. Ces mentions ne servent qu’à miner la crédibilité de la travailleuse par une référence subtile au mythe voulant que seuls les hommes déviants se livrent à des gestes de harcèlement sexuel (Lonsway, Cortina et Magley 2008, 600). La juge emploie d'ailleurs un temps de verbe conditionnel (lui aurait lancé une tasse; aurait crié après elle) pour décrire l'événement culminant invoqué par la travailleuse : «... la travailleuse décrit également une situation où monsieur G... aurait crié après elle, devant des clients. Par la suite, la travailleuse serait allée lui parler dans la cuisine. Monsieur G... lui aurait alors lancé une tasse, que la travailleuse a pu éviter ».

Par la suite, quand la travailleuse entend la voix du harceleur alors quelle est retournée travailler dans un autre établissement dans lequel elle ne s'attendait pas à le voir, la juge rapporte que la travailleuse est " indisposée », comme si elle était mécontentée plutôt qu'apeurée : «Selon la travailleuse, alors qu’elle se trouve au service au volant, elle entend monsieur G... crier dans l'établissement. Ceci l'indispose. Elle demande à retourner à l'établissement 6095 ».

Par contre, lorsque le son de la voix de la travailleuse « indispose » son collègue, la juge devient beaucoup plus éloquente. Elle présente un lien entre l'agacement ressenti par celui-ci, les émotions d'impatience et d'irritation qu'elle provoque chez lui, et la production de son comportement qualifié de " parfois colérique » à l'égard de la travailleuse. Elle emploie l’indicatif présent pour présenter le point de vue du travailleur : «Monsieur G... est agacé par le fait que la travailleuse a tendance à parler fort, voire même crier, ce qui l'indispose. Monsieur G... peut donc démontrer de l'impatience et de l'irritation. C'est ce qui peut expliquer son comportement parfois colérique à l'égard de la travailleuse ». 
Le harceleur est dépeint comme quelqu'un qui se retient plutôt que comme quelqu'un qui s'emporte. Le binôme victime/harceleur est, encore une fois, inversé.

En fin de compte, la juge rejette la réclamation. Elle conclut à un « conflit interpersonnel ayant mal évolué et auquel s'est ajouté une perception subjective de la part de la travailleuse " [nos italiques]. Ce faisant, la juge greffe un ultime binôme à sa logique binaire, soit le binôme privé (conflit interpersonnel)/public (conflit régi par la LATMP). Elle laisse entendre que les perceptions subjectives de la travailleuse émotive et irrationnelle appartiennent à la sphère privée des relations interpersonnelles, perpétuant ainsi le mythe voulant que, même si le harcèlement sexuel se produit dans la sphère publique, celui-ci demeure une affaire personnelle (Wood 2005).

\section{Comportement contraignant? Ou simplement contrariant...}

Dans la décision D.C. et Compagnie A (2014 QCCLP 330), le travailleur allègue qu'il s'est fait « empoigner » les fesses et les pectoraux par son superviseur qui « lui parlait souvent de sexe au travail ». Ce dernier admet par ailleurs avoir donné une tape sur les fesses du travailleur alors que celui-ci était penché et lui faisait dos. Dans sa réclamation, le travailleur déclare avoir été agressé sexuellement alors qu'il avait dix ans, d’où sa forte réaction aux gestes inappropriés de son superviseur et son diagnostic de trouble d'adaptation. Le travailleur n'est pas présent à l'audience. La juge entend les témoignages du superviseur et d'un conseiller en santé et sécurité du travail qui a procédé à une enquête interne pour le compte de l'employeur.

Dans ces circonstances, encore une fois, que la juge n'ait pas retenu la version du travailleur absent et rejette la réclamation n'a peut-être rien de surprenant. Toutefois, sa décision propose une interprétation de la LATMP voulant que seul un comportement contraignant ou plus, par opposition à un simple comportement contrariant, puisse constituer l'événement imprévu et soudain requis pour la reconnaissance d'une réclamation en vertu de la LATMP, soit un critère qui outrepasse clairement les exigences de la Loi.

La juge se réfère à « une analyse des comportements en matière de harcèlement sexuel selon le degré » suivant un tableau paru dans la revue Relations industrielles en 1988. Le tableau divise les comportements selon leur forme (non verbale, verbale ou physique) et leur degré (contrariants, contraignants ou agressants), indiquant d'un astérisque les comportements qui doivent être répétés pour qu'il y ait harcèlement sexuel. Par exemple, un frôlement ou un tapotement représente un comportement considéré " contrariant »; il doit donc être répété pour être considéré du harcèlement sexuel. Soulever un vêtement est considéré une forme de harcèlement sexuel " contraignant », et ce, même sans répétition. Par ailleurs, c'est seulement si le vêtement est arraché que le harcèlement est considéré " agressant » (Savoie et Larouche 1988).

Cette approche représente l'apogée d'un modèle fonctionnaliste du harcèlement sexuel, un modèle qui aujourd'hui est remis en question (McDonald 2012; Bingham 2005; Dougherty 2004). Une telle approche a comme prémisse que le harcèlement constitue un problème rationnel et ordonné qui peut être vérifié objectivement; la complexité du harcèlement sexuel, sa dimension subjective et ses conséquences sur la victime sont évacuées. 
En adoptant un modèle hyper-fonctionnaliste, la juge tente de produire une apparence de rationalité et de créer une illusion de détachement et de neutralité quant à l'appréciation des événements invoqués par le travailleur. L'effet est une sur-simplification et une dé-contextualisation du harcèlement. En outre, il est fort compréhensible que les expériences passées de la victime du harcèlement sexuel influencent l'impact du harcèlement sur elle (Stockdale et al. 2014), c'est ce qu'affirme, d'ailleurs, le travailleur lorsqu'il invoque l'agression sexuelle qu'il a subi dans son enfance. La fausse logique binaire proposée par la juge - comportement contrariant/comportement contraignant - se mute rapidement en un autre binôme, soit le harcèlement sexuel sans conséquence/le harcèlement sexuel avec conséquences. Cette logique approuve l'idée que seulement le harcèlement sexuel grave - contraignant ou agressant - cause des torts à ses victimes, contrairement aux petits gestes contrariants - de harcèlement sexuel qui ne méritent pas que l'on s'y attarde. Ainsi, la décision D.C. et Compagnie A normalise et banalise le harcèlement sexuel, tout comme les décisions M.H. et Compagnie B et Launière et Pekuakamiulnuatsh Takuhikan.

\title{
Exemples d'analyse globale et contextuelle
}

Heureusement, ce ne sont pas tous les décideurs qui bâtissent les motifs de leurs décisions à partir d'un agencement de logiques binaires. En contraste aux exemples mentionnés ci-hauts, dans la décision Gamache et Société des transports de Montréal (2015 QCCLP 2636), la juge souligne la corroboration du témoignage de la travailleuse mais cette fois-ci, sans miner la valeur probante de celui-ci :

\begin{abstract}
En raison de l'absence de toute condition personnelle et surtout en raison du témoignage de la travailleuse, tout à fait crédible et non contredit par l'employeur, laquelle a expliqué en détail ce qu'elle avait vécu, et aussi, en raison de la preuve documentaire qui corrobore son témoignage, la Commission des lésions professionnelles conclut que...la travailleuse a subi une lésion professionnelle. (Nos italiques)
\end{abstract}

Dans la décision Lapierre et Hôtel Traversy (2015 QCCLP 5641), une travailleuse qui occupait un emploi de serveuse dans un bar a subi du harcèlement sexuel et du harcèlement psychologique de la part du nouveau propriétaire de l'établissement. Même si la juge ne nomme pas le harcèlement sexuel explicitement, elle souligne d'emblée la gravité des gestes constituant du harcèlement sexuel, et ce, avant même de relater d'autres gestes de harcèlement psychologique posés à l'endroit de la travailleuse : «Le tribunal retient de la preuve que l'employeur s'est livré à des contacts physiques non sollicités et non consentis par la travailleuse sur ses fesses et ses seins. Ce seul comportement imprévu et soudain déborde le cadre normal du travail ».

Dans la décision J.D. et Compagnie A (2016 QCTAT 4912), la travailleuse avait, avant les faits reprochés, une relation amoureuse consentante avec son nouveau supérieur. La juge affirme qu'il s'agit « d'une situation qui, dans sa globalité, n’est pas simple, et certains événements décrits sont « limites ». Cela dit, elle situe son analyse du harcèlement sexuel subi par la travailleuse dans le contexte des rapports de pouvoir entre celle-ci et son supérieur : 
Il est vrai que la relation amoureuse qui a débuté entre madame D... et monsieur M... était au départ consensuelle. Et que le milieu de travail a constitué « l'environnement physique » qui a favorisé l'éclosion de cette relation, et cette relation, évidemment d’ordre personnel, était étrangère comme telle au travail exercé par chacun d'eux.

Mais il y a un « mais». Il ne faut cependant pas oublier que monsieur M... a toujours été son supérieur immédiat, avec les pouvoirs et obligations inhérents à cette fonction, dont un pouvoir d’autorité sur la travailleuse...

La juge focalise sur le comportement du harceleur et l'exercice de son pouvoir hiérarchique à l'endroit de la travailleuse pour ensuite conclure que les événements reprochés se sont déroulés dans la sphère professionnelle :

... monsieur M... « utilise » en quelque sorte le travail, voire même son statut hiérarchique pour avoir des propos, des insultes et des gestes dégradants à l'endroit de la travailleuse. Il lui fait des remarques quant à la longueur de ses interventions avec des clients, lui disant même quelle avait des relations sexuelles avec eux et quelle en conservait des traces physiques. Il utilise même le matériel fourni par l'employeur pour lui envoyer des messages textes très agressifs. Lorsqu'il suit la travailleuse, il lui fait des attouchements.

Le cumul des événements conduira ultimement au 20 décembre 2013, alors que monsieur M... utilise de façon évidente son pouvoir hiérarchique pour lui dire qu'elle avait démissionné de son emploi, alors que ce nétait pas le cas. Cela s'est clairement passé dans la sphère professionnelle. ...

Le fait de placer le comportement du harceleur plutôt que la perception de la travailleuse au centre de son analyse lui permet de passer outre le fait qu'en dépit du comportement répréhensible du harceleur, la travailleuse semble néanmoins avoir eu une peine d'amour à la fin de la relation : " Même si le courriel transmis par la suite à madame N... [la présidente de la compagnie] fait état de sentiments provenant d'une femme blessée à la suite d'une relation amoureuse, cela ne transcende pas les gestes et faits posés par monsieur M... à son endroit, qui débordent clairement ce à quoi on peut s'attendre dans un milieu de travail ».

Non seulement la décision n’emploie pas un langage binaire, mais elle écarte le stéréotype de la "victime idéale " d'une agression sexuelle ou du harcèlement sexuel (Robichaud et CCDP 1987).

De façon semblable, dans la décision C.P. et Compagnie A (2014 QCCLP 713), le juge rejette la réclamation, mais sans avoir recours à un langage binaire. Il rappelle que ce sont les règles de preuve qui font que la réclamation est jugée inadmissible, et non une vérité absolue :

En l'instance, la preuve de la travailleuse repose sur son seul témoignage, lequel est contredit par celui de l'employeur. Sans conclure pour autant que l'employeur soit exempt de tout reproche... la crédibilité de la travailleuse est malheureusement entachée par ses nombreux antécédents criminels ainsi que par son omission de révéler ses récents démêlés avec la justice. Celle-ci aurait certainement eu avantage à faire entendre la collègue impliquée dans l'incident pour corroborer son témoignage. Le tribunal rappelle que le fardeau d'établir l'existence d'un accident du travail repose sur la travailleuse. 
Lempathie démontrée par le juge pour la travailleuse et le soin de présenter les faits principalement de sa perspective à elle font que la décision ne véhicule pas de préjugé relatif au harcèlement sexuel, et ce, même si la réclamation de la travailleuse est rejetée.

\section{Conclusion}

Par une argumentation réfléchie et bien ordonnancée, la décision d'un tribunal vise à nous convaincre de la vérité des constats du décideur et du bien-fondé de ses conclusions. Au-delà du sort de la réclamation dont il est question, la présente étude a fait ressortir la portée idéologique des décisions du TAT-DSST en matière de harcèlement sexuel. Elle met en relief le rôle actuel du régime de la LATMP dans la banalisation du harcèlement sexuel au travail et, en même temps, son rôle potentiel dans la reconnaissance des torts causés aux femmes par celui-ci.

Il est de lessence d'une décision rendue par un tribunal d'appliquer une règle de droit aux faits, et, ce faisant, de répondre à certaines « questions caractéristiques » (Van Eemeren et al. 1997) comme, en l'espèce, celles concernant les critères d’admissibilité d'une réclamation en vertu de la LATMP; le droit appelle nécessairement à la catégorisation (Hunter 2015b). Toutefois, au-delà de la catégorisation inhérente au droit, plusieurs décisions du TAT-DSST en matière de harcèlement sexuel font appel à un langage binaire, soit une forme de catégorisation dans laquelle les femmes et les caractéristiques associées aux femmes sont infériorisées (Dougherty et Hode 2016).

La présence de logiques binaires dans une décision portant sur le harcèlement sexuel traduit des attitudes, des valeurs et des croyances dans lesprit du décideur qui ont pu influencer l'ensemble de l'audition et, plus particulièrement, l'appréciation de la preuve, lévaluation de la probité des témoignages, et ultimement, le sort de la réclamation (Hunter, Roach Anleu et Mack 2016). Par conséquence, les résultats de notre recherche appellent à une rectification de la situation pour les travailleuses harcelées sexuellement au travail dont le recours exclusif est une réclamation en vertu de la LATMP.

En effet, nos résultats ont démontré que, par l'emploi d'un langage binaire, le discours décrypté dans la moitié des décisions analysées renvoie à des attitudes et à des croyances qui servent à nier, voire à justifier le harcèlement sexuel des femmes.

Ces attitudes et croyances veulent notamment que :

- Les femmes harcelées sexuellement soient les auteures de leur propre malheur.

- Ce sont des hommes déviants qui posent des gestes de harcèlement sexuel (les hommes ordinaires n'en posent pas).

- Les femmes déposent des plaintes pour harcèlement sexuel par esprit de vengeance, pour attirer l'attention ou pour cacher leur propre inconduite.

- Les femmes qui se disent harcelées sexuellement au travail exagèrent.

- Les femmes qui attendent ou hésitent à dénoncer le harcèlement sexuel inventent les faits rapportés, ou encore, les faits ne sont pas très sérieux (Lonsway, Cortina et Magley 2008).

Largumentation dans les décisions du TAT-DSST peut donc être vue comme un mécanisme de régulation du discours (Van Eemeren et al. 1997) relatif au 
harcèlement sexuel. Autrement dit, indépendamment du sort qu'elle réserve à la réclamation, une décision est susceptible, soit de perpétuer des attitudes et des croyances qui servent à banaliser le harcèlement sexuel, soit de les remettre en question (Marshall 2005) et ce, dans un cadre juridique formel identique.

En outre, un langage binaire fait en sorte que certaines décisions vont même interpréter la LATMP de façon à créer une catégorie juridique du harcèlement sexuel considéré comme étant sans conséquence pour les victimes, soit une ultime banalisation du harcèlement sexuel. Le droit de la travailleuse à un milieu de travail exempt de harcèlement sexuel est bafoué, et ce, en toute impunité pour l'employeur et pour le harceleur, avec en prime le sceau d'approbation du TAT-DSST. Pas étonnant, dans ce contexte, que les femmes hésitent à dénoncer le harcèlement sexuel au travail (Marshall 2005).

Par ailleurs, sur une note plus positive, nos résultats démontrent également que plusieurs décisions ne tombent pas dans le piège des logiques binaires et des stéréotypes à l'égard des femmes qui allèguent un problème de santé en lien avec le harcèlement sexuel au travail. Cela suggère que la conscientisation des juges à leurs propres valeurs - conscientes et inconscientes - est une mesure importante pour s'assurer que les décisions du TAT-DSST évitent systématiquement de mobiliser des arguments qui servent à banaliser le harcèlement sexuel des femmes au travail.

\section{Références}

American Psychiatric Association (APA). 2000. Diagnostic and Statistical Manual of Mental Disorders IV (DSM-IV). Washington, D.C.: American Psychiatric Association.

Bernheim, Emmanuelle. 2013. Prendre le droit comme un «fait social» - La sociologie du droit par et pour elle-même. Dans Les cadres théoriques et le droit - Actes de la $2^{e}$ Journée d'étude sur la méthodologie et l'épistémologie juridique. Cowansville : Yvon Blais.

Bingham, Shereen (dir.). 2005. Conceptualizing Sexual Harassment as Discursive Practice. Westport/Londres : Praeger.

Commission des droits de la personne et des droits de la jeunesse (CDPDJQ). 2015. Commentaires relatifs à la consultation portant sur le rapport de mise en cuvre du Plan d'action gouvernemental en matière d'agression sexuelle (Cat. 2.115.56). <http:// www.cdpdj.qc.ca/Publications/commentaires plan action agression sexuelle.pdf>

Conley, Robin, et William M. O’Barr. 1998. Just Words: Law, Language, and Power, $2^{\mathrm{e}}$ éd. Chicago/Londres : University of Chicago Press.

Corbin, Juliet, et Anselm L. Strauss. 2008. Basics of Qualitative Research, $3^{\mathrm{e}}$ éd. Thousand Oaks (Californie) : Sage Publications.

Cox, Rachel. 2014. From sexual to psychological harassment: One step forward, twentyfive years back for women's equality at work? Dans 14 Arguments in Favour of Human Rights Institutions, Shelagh Day, Lucie Lamarche et Ken Norman, dir. Toronto : Irwin Law.

Cox, Rachel. 2015. Le harcèlement pour un motif interdit en droit québécois : la captation de la norme de la Charte par la panoplie de régimes applicables dans les milieux de travail (Conférence, Centre de recherche interuniversitaire sur la mondialisation et le travail Montréal, 21 mai 2015).

Dougherty, Debbie, et Marlo Hode. 2016. Binary Logics and the discursive interpretation of organizational policy: Making meaning of sexual harassment policy. Human relations 69 (8): 1729-55. 
Dougherty, Debbie, et Mary Jeanette Smythe. 2004. Sensemaking, Organizational Culture and Sexual Harassment. Journal of Applied Communication Research 32 (4): 293-317.

Fairclough, Norman. 2010. Critical Discourse Analysis: The Critical Study of Language, $2^{\mathrm{e}}$ éd. Londres/New York : Routledge.

Fudge, Judy. 2013. From Women and Labour Law to Putting Gender and Law to Work. Dans The Ashgate Research Companion to Feminist Legal Theory, Margaret Davies et Vanessa E. Munro, dir. Farnham : Ashgate.

Hunter, Rosemary, Sharyn Roach Anleu et Kathy Mack. 2016. Judging in lower courts: Conventional, procedural, therapeutic and feminist approaches. International Journal of Law in Context 12 (2): 337-60.

Hunter, Rosemary. 2015a. Feminist judgments projects: Legal fiction as critique and praxis. International Critical Thought 5:501-508.

Hunter, Rosemary. 2015b. More than just a different face? Judicial diversity and decision-making. Current Legal Problems 68:119-41.

Lazar, Michelle M. 2007. Feminist critical discourse analysis: Articulating a feminist discourse praxis. Critical Discourse Studies 4 (2): 141-64.

Langevin, Louise. 2005. Progrès ou recul : réflexions sur l'accessibilité à la justice pour les victimes de harcèlement sexuel au Québec. Revue Femmes et Droit 17:197-217.

Lippel, Katherine, et Rachel Cox. 2010. Droit de la santé au travail régissant les problèmes de santé mentale : prévention, indemnisation et réadaptation. Dans JurisClasseur Québec, Feuilles mobiles, Collection Santé et Sécurité du Travail, Fascicule 27, LexisNexis Canada. Dernière mise à jour novembre 2016.

Lippel, Katherine, Rachel Cox et Isabelle Aubé. 2009. Interdiction du harcèlement et protection de la vie privée et des droits fondamentaux. Dans JurisClasseur Québec, Feuilles mobiles, Collection Travail, Fascicule 23, LexisNexis Canada. Dernière mise à jour juin 2018.

Lippel, Katherine, et Diane Demers. 2000. Le harcèlement sexuel au travail : la rencontre du droit de la santé au travail et des droits de la personne. Canadian Journal of Women \& Law 12 (1): 32-65.

Livholts, Mona. 2015. Discourse, power and representation. Dans Discourse and Narrative Methods: Theoretical Departures, Analytical Strategies and Situated Writings, Mona Livholt et Maria Tamboukou, dir. Londres : SAGE.

Lonsway, Kimberly A., Lilia M. Cortina, et Vicki J Magley. 2008. Sexual harassment mythology: Definition, conceptualization, and measurement. Sex Roles 58:599-615.

MacKinnon, Catharine. 1979. Sexual harassment of working women: A case of sex discrimination. Londres : Yale University Press.

Makela, Finn. 2006. “Tell me where it hurts": Workplace sexual harassment compensation and the regulation of hysterical victims. McGill Law Journal 51:27-46.

Marshall, Anna-Maria. 2005. Confronting Sexual Harassment. Aldershot (R.-U.) : Ashgate.

McDonald, Paula. 2012. Workplace sexual harassment 30 years on: A review of the literature. International Journal of Management Reviews 14:1-17.

Nightingale, A., M Quayle et O Muldoon. 2017. "It's just heart breaking”: Doing inclusive political solidarity or ambivalent paternalism through sympathetic discourse within the "refugee crisis" debate. Journal of Community and Applied Social Psychology 27:137-146.

Olsen, Frances. 1998. The Sex of Law. Dans The Politics of Law. A Progressive Critique, 3 e éd., David Kairys, dir. New York : Basic Books.

Paquet, Jean-Claude. 1997. Laffaire Béliveau-St-Jacques : Léquilibre entre l'intégrité du régime de réparation des lésions professionnelles et le droit à des dommages exemplaires en vertu de la Charte des droits et libertés de la personne. Dans Développements récents en droit de la santé et sécurité du travail. Cowansville: Yvon Blais. 
Reiner, Günter. 2016. Les dichotomies en droit. Dans Les nouveaux chantiers de la doctrine juridique. Actes des $4^{e}$ et $5^{e}$ journées détude sur la méthodologie et lépistémologie juridiques, Georges Azzaria, dir. Montréal : Yvon Blais.

Savoie, Dominique, et Viateur Larouche. 1988. Le harcèlement sexuel au travail : définition et mesure du phénomène. Relations industrielles 43:509-24.

Stockdale, Margaret S., T. K. Logan, Katherine A. Sliter et Seth A. Berry. 2014. Interpersonal violence victimization and sexual harassment: A prospective study of revictimization. Sex Roles 71 (1-2): 55-70.

Van Eemeren, Frans H., Rob Grootendorst, Sally Jackson et Scott. Jacobs. 1997. Argumentation. Dans Discourse as Structure and Process. Discourse Studies: A Multidisciplinary Introduction, Teun A. Van Dijk, dir. Londres : SAGE.

Wood, Julia T. 2005. Saying it makes it so, the discursive construction of sexual harassment. Dans Conceptualizing Sexual harassment as Discursive Practice, Shereen Bingham, dir. Westport/Londres : Praeger.

\section{Législation citée}

Loi sur les accidents du travail et les maladies professionnelles (LATMP), RLRQ c A-3.001

Loi sur les normes du travail (LNT), RLRQ c N-1.1

Projet de loi 176, Loi modifiant la Loi sur les normes du travail et d'autres dispositions législatives afin principalement de faciliter la conciliation travail-famille, LQ 2018, c. 21.

\section{Jurisprudence citée}

Janzen c. Platy Enterprises ltd, [1989] 1 RCS 1252.

Québec (Commission des droits de la personne et des droits de la jeunesse) c. Québec (Procureur général), [1998] RJQ 3397.

Robichaud et Commission canadienne des droits de la personne, [1987] 2 RCS 84.

\section{Décisions analysées}

T.T. et Compagnie A, 2014 QCCLP 269

D.C. et Compagnie A, 2014 QCCLP 330

C.P. et Compagnie A, 2014 QCCLP 713

M.V. et Compagnie A, 2014 QCCLP 1981

K.K. et Commission scolaire A, 2014 QCCLP 2690

Gamache et Société des transports de Montréal, 2015 QCCLP 2636

Lapierre et Hôtel Traversy, 2015 QCCLP 5641

Violi et Traiteur Dine-Aire, 2015 QCCLP 6867

J.D. et Compagnie A, 2016 QCTAT 4912 M.H. et Compagnie B, 2016 QCTAT 3846

Launière et Pekuakamiulnuatsh Takuhikan, 2016 QCTAT 4968

M.N. et Compagnie A, 2016 QCTAT 5227

Rachel Cox

Département des sciences juridiques

Université du Québec à Montréal

Montréal (Québec)

cox.rachel@uqam.ca 